\title{
Interference Aware Priority Based Packet Forwarding In Wireless Sensor Network Using Bluetooth
}

\author{
Abdul Ali, M. Vadivel
}

\begin{abstract}
Wireless Sensor Networks consists of several nodes that are distributed over a particular area. These sensor nodes are able to sense the changes in environmental parameters like temperature and carbon monoxide. Depending upon the ability each sensor node possess the type of wireless sensor networks may vary: that is either Homogeneous or Heterogeneous. This particular paper is concentrated on a homogeneous network. In this paper, an Interference Aware Priority based Packet Forwarding in Wireless Sensor Network using Bluetooth (IAPFB) scheme is proposed which helps in the congestion control in Wireless Sensor Network. The main idea behind this paper is that ; avoid the interference and collision between the nodes in the network while transmitting the data packets and also the higher priority data must forwarded first than a low priority one. The main application of this concept is the Body Sensor Networks. That is the body sensors for grabbing the signals from various body parts is used as the operating network. Signals from different body parts may have different priority levels and the proposed scheme can easily deal with the priorities. Simulated results shows comparatively good results for the proposed method.
\end{abstract}

Keywords-Wireless Sensor Networks (WSNs), Wireless Body Sensor Networks (WBSNs), Body Sensor Networks (BSNs), Healthcare Systems.

\section{INTRODUCTION}

$\mathrm{W}$ ireless Sensor Network (WSN) is defined as a group of dedicated sensors that are spatially distributed for monitoring and recording the environmental changes and forward the gathered data to the base station. In a WSN, a sensor node may have multiple sensor for capturing different types of data. In the large scale applications of WSNs, such as agricultural scenarios or military applications a large number of nodes are deployed over a wide coverage area. All the nodes in this network collects data from its range and send them to base station as soon as possible. In these scenarios, large number of nodes became active and create heavy traffic. Although large amount of sensed data is good for maximizing throughput so that all the nodes can transmit them as quickly as possible to save energy, it may cause congestion in the network.

Revised Manuscript Received on October 01, 2019.

* Correspondence Author

Abdul Ali*, Department of Computer Science and Engineering, Sathyabama Institute of Science and Technology, Chennai, India. Email: abdulalikm86@gmail.com

Dr. M. Vadivel*, Department of Electronics and Communication Engineering, Vidya Jyothi Instituite of Technology, Hyderabad, India. Email: drmvadive179@gmail.com
In such applications, the network follows tree or mesh topology. Congestion in network can have a negative impact on the performance, throughput, packet delivery and energy. And also the fact of being in the same wireless coverage of each other leads to communication interference. Traffic in Wireless Sensor Networks follows a centralized pattern, thus the idea of bypassing hot spot would not reduce congestion, since it will definitely appear again somewhere near the sink.

In real time scenario, packets collision due to link interference or packet loss due to congestion on inter or intra path nodes eventually affect the application throughput as well as energy consumption. Hence, in such applications Congestion Control mechanisms has greater importance. Here the Congestion control scheme is a two-step procedure: 1) congestion and interference detection 2) Rate control mechanism establishment.

In this research work, our aim is to build a scheme that can handle the packet collisions or packet loss due to interference or congestion and give more importance to packets with high priority during the scheduling. Here, we concentrate on Wireless Body Sensor Networks (WBSNs) in Health care systems. Monitoring human body using WSN can be achieved by attaching sensors to the body surface or implanting them into tissues. And since the proprietary designed WSNs are not suited for monitoring the human body led to the development of Wireless Body Sensor Networks (WBSNs). Monitoring a patient also includes the vital sign measurements such as blood pressure, temperature, heart rate. For hospitals where many patients are treated every year Body Sensor Networks (BSNs) offers special benefits.

\section{RELATED WORK}

Congestion Control is one of the most important challenge that Wireless Sensor Networks faces. The reason for congestion in each network may vary such as buffer overflow, collision of packets, interference, transmission rate, transmission channel problems. And hence the various existing methods deals with different parameters of congestion. Event to Sink Reliable Transport (ESRT) [9] is a congestion control scheme which supports a centralized rate allocation. This protocol is operated based on the events occurring in the network and that's why it is called an event driven protocol. Here the sending rates for all nodes are controlled by sink and all the nodes are assumed to be situated at one hop distance from sink. In this work the event reliability is defined as the number of packets that should be transmitted from all the nodes for reliable event detection.

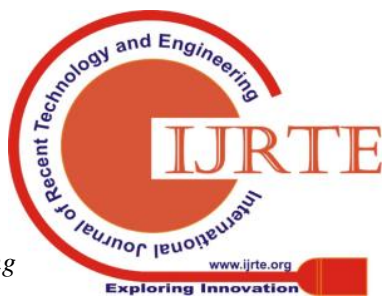


All the nodes sends packets at a fixed rate until the sink send them any updates. Sink computes the rate updation by using the congestion level and good put. Although the scheme minimizes energy utilization and ensure fairness, throughput achieved is not efficient.

In FLUSH [10], the large data is divided into a number of packets and they are transmitted in a pipelined manner. Flush is a reliable, receiver initiated bulk data transport protocol for Wireless Sensor Networks. It provides end to end reliability and reduces the transfer time. Flush assumes that only one source (flow) is active at a time for a sink and it uses ACKs for ensuring reliability and hop by hop rate control. To initiate the data transmission, sink sends request for an object to a particular source and followed by four phases: topology query, data transfer, acknowledgement and integrity check.

Delay Aware Priority based Routing (DAPR) [6] scheme describes the ways to deals with data packets with different priority. The data sensed by two nodes may have two priority levels and hence these two packets must handle separately. This scheme has two priority levels: High priority (HP) data and Low priority (LP) data. Each node in the network is assigned a unique id and the buffer has two queues one for HP data and one for LP data. Differentiator filters the incoming packets and forwarded to the respective queue, while the scheduler schedules the data packets in which the packets in HP queue scheduled first.

Interference Aware Congestion Control (IACC) [5] achieves maximum link utilization by avoiding the interference and thereby controlling congestion. This protocol concentrates on interference and collision between nodes during data transfer. For each node to be congestion free, the amount of data that is send should be greater than the amount of data received and generated. And for avoiding collision, no two nodes who are within the transmission range of each other can send packets to sink during the same slot.

Reliable Efficient Fair Interference Aware Congestion Control (REFIACC) [7] scheme is a modification of the IACC scheme. This paper tries to solve the throughput maximization problem using an efficient congestion control based scheduling algorithm along with REFIACC protocol. It avoids the interference and achieves higher bandwidth utilization and throughput. Interference is controlled by considering the difference in the link capacities during the process of scheduling. And linear programming for maximum bandwidth utilization. The simulation results shows its efficiency in higher packet reception ratio and throughput compared to other literature work such as TARA and FLUSH.

\section{NETWORK MODEL}

Here we are assuming that each and every node in the network have synchronized clock and a half-duplex radio transceiver. The communication between nodes can be represented by an undirected graph $\mathrm{G}=(\mathrm{V}, \mathrm{E})$, where $\mathrm{V}$ indicates the set of nodes and $\mathrm{E}$ is the set of edges. Every edge $(a, b) € E$ is supposed to having a capacity $\mathbf{C}_{\mathbf{a}, \mathbf{b}}$

The data are gathered over a tree structure $\mathrm{G}_{\mathrm{T}}=\left(\mathrm{V}_{\mathrm{T}}, \mathrm{E}_{\mathrm{T}}\right)$ rooted at base station $B_{s}$. However the real problem is how the data can be gathered without collision and congestion

through the tree structure. And in this paper we try to untackle the same problem and maximizing the network throughput. All nodes in the network continually generate data. And each node assigned time frame for transmission.

Time is divided into fixed length contiguous frames, and each frame constitutes a set of slots having same duration $\underline{\text {. }}$. During each slot data packets are transmitted from set of children nodes to their parents. $\mathrm{F}(\mathrm{a})$ denotes the frame allocated for node ' $a$ ' to generate/ receive/ transmit data.

To maximize the throughput and prevent the collision and congestion, the nodes should satisfy the following constraints:

1. Only source nodes generate data

2. The data transmission rate from ' $a$ ' to its parent ' $b$ ' should not exceed the link capacity $\mathrm{C}_{\mathrm{a}, \mathrm{b}}$

3. No node can send and receive data simultaneously.

4. No two nodes can transmit at the same slot if both are within each other's transmission range.

5. Ensure that for each node the amount of transmitted data should be greater than the amount of received and generated data.

6. If any node encounters some high priority data, it should be transmitted as soon as possible.

\section{INTERFERENCE AWARE CONCEPT}

Implementation of Interference Aware concept is a two-step procedure. First we have to estimate the link capacity between each pair of node and forward the same to Base station/ Sink. In the second step, a proper scheduling is established to ensure the fairness and data priority.

\section{A. Estimating Link Capacity}

In this interference aware approach, Congestion control is based on the original link capacity. For estimating the link capacity, each node 'a' sends a burst of packets to its parent ' $b$ ' during a time period T. Note that no new packet will be sent until receiving the acknowledgement of previous packet. The link capacity of nodes can then computed by total number of acknowledged packets divided by the time period T. Once this step is over, nodes forward their capacities along with its parent ID to base station.

\section{B. Schedule Construction}

Schedule construction phase is done by the base station upon collecting the link capacities from nodes in the communication tree. For constructing a proper schedule, every node ' $a$ ' sets an appropriate sending rate and this should not exceed the capacities of router nodes in the path to base station.

To ensure the fairness base station must find the total number of source nodes in the sub tree of node ' $a$ ' and is represented as $\mathrm{N}_{\mathrm{a}}$. Thus node ' $\mathrm{a}$ ' can sent at least $\mathrm{N}_{\mathrm{a}}$ packets during a frame. Because the frame is divided into multiple slots with duration $\delta$, node ' $\mathrm{a}$ ' is assigned a frame with $\mathrm{S}_{\mathrm{a}}=\left[\mathrm{N}_{\mathrm{a}} / \delta \mathrm{C}_{\mathrm{a}, \mathrm{b}}\right]$ where ' $b$ ' is parent of ' $a$ '. During the above computation, if the reminder happens to be not null then the last assigned slot will not be fully used. 
The remaining slot can be fairly divide between the nodes in the sub tree of ' $a$ '.

\section{Slot Assignment}

Now that we have number of slots per frame needed by each node, can start the slot assignment process. Our aim is to avoid collisions due to interference and hence we allow the non-interfering links to transmit simultaneously. For that we first built an interference graph $\mathrm{G}_{\mathrm{I}}=\left(\mathrm{V}_{\mathrm{I}}, \mathrm{E}_{\mathrm{I}}\right)$. The set of vertices $V_{I}=E_{T}$ represent the set of links between the nodes and their parents in the tree structure. Two links, say $(p, q)$ and $(r, s)$ are connected in $G_{I}$ if they are in interference with each other.

The slot assignment algorithm is an iterative process. In every iteration $n$, it tries to find out the maximal independent set of vertices say $I_{n}$, with higher number of edges. This in turn cuts the interference graph into several sub graphs and that helps in reusing slots fastly. Since the radio links in $I_{n}$ are not interfering, the set of nodes $\left\{a \mid(a, b) \in I_{n}\right\}$ can transmit in slot $\mathrm{n}$. Once a slot is allocated to a node ' $\mathrm{a}$ ', it decreases the number of slots required for each frame. And whenever the required slots reaches zero, all the vertices that are connected with node 'a' and their respective links from graph $\mathrm{G}_{\mathrm{I}}$ should be removed immediately. The above process repeats until the graph $\mathrm{G}_{\mathrm{I}}$ becomes empty.

\section{Bluetooth Protocol And Concept}

A lot of electronic devices such as mobile phones, headsets, laptops and vehicle uses Bluetooth technology. And the main advantage of this particular technology is that it allows hands-free communication between devices. That means even if the user is out of coverage of a hot spot or broadband connection, he/she can get access to the Internet for a while. Bluetooth is a wireless technology standard for exchanging data over short distances and it uses UHF radio waves which is in the ISM band 2.4 to $2.485 \mathrm{GHz}$. The bluetooth devices forms a Personal Area Network (PAN) for communicating with each other. Initially Bluetooth was standardized with IEEE 802.15.1 but it no longer maintains its standard.

Bluetooth has 79 designated channels for transmitting data packets each of $1 \mathrm{MHz}$ bandwidth. When a Bluetooth device needs to transmit data, it splits that data into a number of packets and each packet is transmitted through any one of the 79 bluetooth channels. And hence we can say that bluetooth is a packet based protocol that follows master/slave architecture. One Bluetooth device can communicate with 7 other bluetooth devices. And there by these 8 bluetooth devices can together form cluster called piconet. Among these 8 nodes, one node will act as master and all others are slaves. That is a pico net consist of one master and 7 slaves. But all the 8 devices share master's clock. The packet exchange is based on the clock defined by master. Master node transmit only in even slots and receives in odd slot. Likewise slave transmit in odd slot and receives in even slots. A number of piconets can be interconnected with bridge nodes to form a large network known as scatter net.

For implementing Bluetooth protocol in NS2 we use the UCBT module. UCBT (University of Cincinnati - Bluetooth) is an NS2 based Bluetooth network module which simulates the Bluetooth network operations. UCBT includes most of the protocols of bluetooth protocol stack such as LMP, L2CAP, BNEP. It includes the functionalities such as connection request, connection establishment, scanning for finding nearby devices, hold, sniff.

\section{Performance Analysis}

In this section the protocol is evaluated using NS2 simulator. The number of nodes are varied up to $2-20$. We performed the analysis based on the following parameters:

TABLE I. SIMULATION PARAMETERS

\begin{tabular}{|l|l|l|}
\hline \multirow{2}{*}{$\begin{array}{l}\text { Sl. } \\
\text { No }\end{array}$} & \multicolumn{2}{|c|}{ Simulation Parameters } \\
\hline 1 & Sarameter & \multicolumn{1}{|c|}{ Value } \\
\hline 2 & $\begin{array}{l}\text { Number of base-station } \\
\text { Nodes }\end{array}$ & 1 \\
\hline 3 & Number of Sensor Nodes & $2-20$ \\
\hline 4 & MAC Protocol & $\begin{array}{l}\text { Bluetooth MAC } \\
\text { protocol }\end{array}$ \\
\hline 5 & Channel Bandwidth & Max. 1MHz \\
\hline
\end{tabular}

1. Throughput

The average throughput of the base station is the amount of packets received successfully at base station during a unit time (packets per seconds). Fig.1 shows the obtained throughput after simulation. The graph (i.e; result.txt) clearly shows that average throughput at the base station improves from time to time than the existing system throughput (sys1).

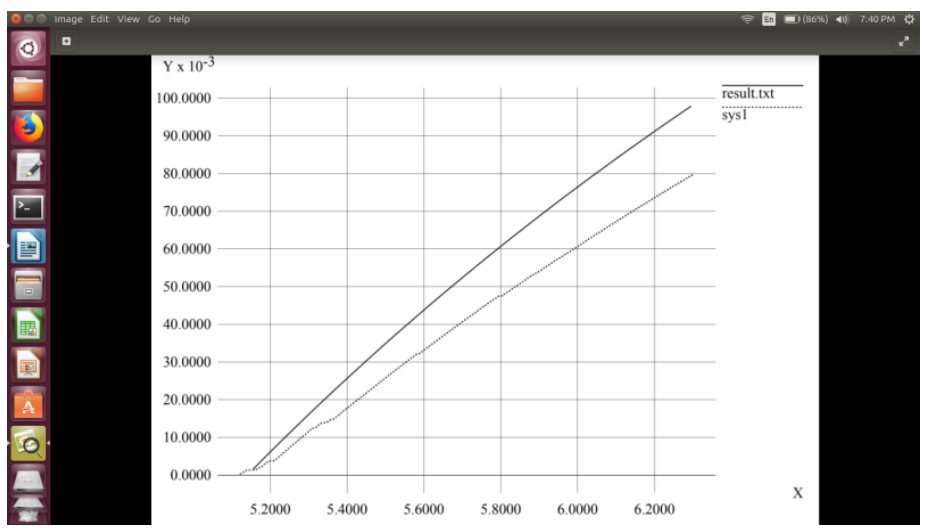

Fig. 1.Simulated Throughput Comparison.

\section{Packet reception ratio}

The average packet reception ratio is the ratio of number of packets received at the base station to the total number of packets generated at source nodes. As we run the simulation, it is seemed that the average packet reception ratio is improved $60 \%$ than the existing systems. Even though the ratio goes low at the starting stage of congestion, it is regained by the congestion control concept of the system. 3. Delay

Delay is an important performance analysis parameter and is defined as how long it takes to reach a packet from source to base station. Since this work includes the priority concept, the value varies according to its priority level. 
However the average delay is seemed to be far better than the existing ones such as TARA and FLUSH. The average end to end delay obtained after simulation is $280.827 \mathrm{~ms}$ and is comparatively a good result for a new concept.

\section{CONCluSION AND FUTURE WORK}

This study discuss about an interference aware priority based packet forwarding using the Bluetooth technology in WSN. To avoid the chances of congestion in the network we use the interference aware concept, then for the urgent transport of emergency messages use the priority concept. This concept can be implemented in the Body Sensor Network as an application. For providing more security and convenience we can use the Bluetooth technology in this particular application. The simulated results showed comparatively better throughput, packet reception ratio, end to end delay. We are planning to implement the concept in Body Sensor Networks with some more advancements and features so that common people can get the advantages of the emerging technologies and concepts.

\section{REFERENCES}

1. Deniz, F., Bagci, H., Korpeoglu, I., \&Yazıc1, A. (2016). “An adaptive, energy-aware and distributed fault-tolerant topology-control algorithm for heterogeneous wireless sensor networks". Ad Hoc Networks, 44, 104-117.

2. Kang, J., Zhang, Y., Nath, B.. "TARA: Topology-Aware Resource Adaptation to Alleviate Congestion in Sensor Networks". IEEE Trans Parallel Distrib Syst 2007;18(7):919-931.

3. Antoniou, P., Pitsillides, A., Blackwell, T., Engelbrecht, A.,\& Michael, L. (2013). "Congestion control in wireless sensor networks based on bird flocking behavior". Computer Networks, 57(5), 1167-1191.

4. C.-Y. Wan, S. B. Eisenman, and A. T. Campbell, "Fuzzy Based Adaptive Congestion control in Wireless Sensor Networks" in Proceedings of the 1st international conference on Embedded networked sensor systems, ser. New York, NY, USA: ACM, 2003 pp. 266-279.

5. Kafi, M. A., Djenouri, D., Othman, J. B., Ouadjaout, A., Bagaa, M., Lasla, N., \&Badache, N. (2014a). "Interference- aware congestion control protocol for wireless sensor networks". Procedia Computer Science, 37, 181-188.

6. Shuvajyoti Pal, and Indrajit Banerjee, "DAPR: Delay-Aware Priority based Routing Scheme to Alleviate Congestion in Wireless Sensor Networks", in 4th International Conference on Information Technology, 2015.

7. M. A. Kafi, J. Ben-Othman, A. Ouadjaout, M. Bagaa, and N. Badache, "REFIACC: Reliable, efficient, fair and interference-aware congestion control protocol for wireless sensor networks," Comput. Commun., vol. 101, pp. 1-11, Mar. 2017.

8. V. Firoiu, M. Borden, "ReRMAC Congestion Control Protocol for Wireless Sensor Networks", in Proceedings of International Conference ofthe IEEE Computer and Communication Societies, 3 (2000)1435-1444.

9. Zgr, Y.S., Sankarasubramaniam, Y., Akan, "O.B., Akyildiz, I.F.. "ESRT: Event-to-Sink Reliable Transport in Wireless Sensor Networks". In Proc. 4th ACM international symposium on Mobile ad hoc networking and computing, MobiHoc. 2003, p. 177-188.

10. Kim, S., Fonseca, R., Dutta, P., Tavakoli, A., Culler, D., Levis, P., et al. "Flush: a reliable bulk transport protocol for multihop wireless networks". In: Proc. of the 5th international conference on Embedded networked sensor systems, SenSys. 2007, p. 351-365.

11. N. Thrimoorthy and Dr. T .Anuradha "A Review on Congestion control Mechanisms in Wireless Sensor Networks ", inInt. Journal of Engineering Research and Applications, November 2014.

12. Wang, C., Sohraby, K., Li, B., Daneshmand, M., \& Hu, Y.(2006). "A survey of transport protocols for wireless sensor networks". IEEE network, 20(3), 34-40.

13. Bian, F., Rangwala, S., Govindan, R.. "Quasi-static Centralized Rate Allocation for Sensor Networks". In: Proc. of 4th Annual IEEE Communications Society Conference on Sensor, Mesh and Ad Hoc Communications and Networks, SECON. 2007, p. 361-370.
14. Hussain, F.B., Cebi, Y., Shah, G.A.. "A multievent congestion control protocol for wireless sensor networks" in EURASIP J Wirel Commun Netw 2008;:44:1-44:12.

15. Ahlam Saud Althobaiti and Manal Abdullah, "Energy Efficient with Collision Free MAC Protocol for Wireless Sensor Network", in Journal of Telecommunication, Electronic and Computer Engineering, 08May 2017, e-ISSN: 2289-8131 Vol. 9 No. 1-3

16. Sara Ghanavati, Jemal Abawajy and Davood Izadi, "A Fuzzy Technique to Control Congestion in WSN", in IEEE Journal,9 Aug. 2013

17. C. T. Ee and R. Bajcsy, "Congestion control and fairness for many-toone routing in sensor networks", in Proc. of ACM International Conference on Embedded Networked Sensor Systems (SenSys '04), pp. 148-161, November 2004.

18. Taj Rahman Siddiqi, Huansheng Ning, Haodi Ping And Zahid Mahmood, "DPCA: Data Prioritization and Capacity Assignment in Wireless Sensor Networks", in 2169-3536 2016 IEEE. Translations, 2017. Volume 5, 2017

19. Sepideh Rowhani Sovaldi, and Mohammad Hosein Yaghmaee Moghaddam, "Fuzzy TADR: A Fuzzy Extension to TADR Algorithm for Congestion Control in Wireless Sensor Network", in IEEE Journal, 2014.

20. Jitesh P. Dhonde and Pankaj A. Salunkhe, "New Approach for Congestion Control Protocol for Wireless Sensor Networks", in International Journal of Advance Electrical and Electronics Engineering (IJAEEE), Volume-5 Issue-2, 2016.

\section{AUTHORS PROFILE}

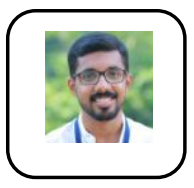

Abdul Ali is a Research Scholar in Computer Science and Engineering at Sathyabama Institute of Science and Technology, Chennai, India. He is having 9 years of experience in teaching. He received his B.Tech. Degree from M.G. University, Kerala, India. He received his M.Tech. Degree from M.S. University, Tirunelveli, India. He has published more than 10 papers in National and International Conference/Journals. His research interest includes Wireless Communication, Wireless Sensor Network.

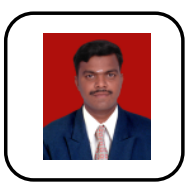

Dr.M.Vadivel is an Associate Professor in Electronics and Communication Engineering at Vidya Jyothi Institute of Technology, Hyderabad, Telangana, India. He is having 16 years of experience in teaching. He received his B.E. Degree from University of Madras, India. He received his M.E and Ph.D. degree from Sathyabama University, India. He has published more than 40 papers in National and International Conference/Journals. His research interest includes Wireless Communication, Wireless Sensor Network, Digital Image Processing and VLSI Design. 